\title{
Schisandra A ameliorates cigarette smoke extract and lipopolysaccharide-induced oxidative stress in lung epithelial cells
}

\author{
Dapeng Zhang ${ }^{1 *}$, Suzhen $\mathrm{Hu}^{2 *}$, Wanyan $\mathrm{Li}^{1}$, Ran $\mathrm{Ao}^{1}$, Zhijuan $\mathrm{Wu}^{1}$, Zhimin Zhang ${ }^{1}$, Ling $\mathrm{Han}^{3}$ \\ ${ }^{1}$ First Affiliated Hospital of Guangzhou Medical University, Guangzhou 510120, China; ${ }^{2}$ Huangdao District Chinese Medicine Hospital, Qingdao \\ 266000, China; ${ }^{3}$ Guangdong Provincial Academy of Chinese Medical Sciences, Guangzhou 510120, China \\ Contributions: (I) Conception and design: Z Zhang, L Han; (II) Administrative support: None; (III) Provision of study materials or patients: None; \\ (IV) Collection and assembly of data: D Zhang, S Hu; (V) Data analysis and interpretation: None; (VI) Manuscript writing: All authors; (VII) Final \\ approval of manuscript: All authors. \\ "These authors contributed equally to this work. \\ Correspondence to: Zhimin Zhang. First Affiliated Hospital of Guangzhou Medical University, Guangzhou 510120, China. \\ Email: zhangzhimin51666@163.com; Ling Han. Guangdong Provincial Academy of Chinese Medical Sciences, Guangzhou 510120, China. \\ Email: linghan36@163.com.
}

Background: The previous studies reported the antioxidant and anti-inflammatory properties of Schisandrin A (Sch A). This study aimed to investigate the ability of Sch A to protect against lung oxidative stress induced by the combination of cigarette smoke extract and lipopolysaccharide (LPS) in an in vitro model of chronic obstructive pulmonary disease (COPD).

Methods: The cell viability was determined by using the 3-(4,5-dimethylthiazol-2-yl)-2,5diphenyltetrazolium bromide (MTT) assay. Colorimetry was used to detect the changes in antioxidant markers. Quantitative real-time polymerase chain reaction (RT-PCR) was used to examine the mRNA levels of interleukin-8 (IL-8) and heme oxygenase-1 (HO-1). The levels of IL-8 and HO-1 in the supernatant were determined by enzyme-linked immunosorbent assay, and Western blot analysis was performed to measure the phosphorylation and protein expression levels of nuclear factor- $\kappa \mathrm{B}$.

Results: Sch A inhibited the excessive proliferation of pulmonary epithelial cells, decreased malondialdehyde content, and increased the expression levels of superoxide dismutase and glutathione after the combined treatment of cigarette smoke extract and LPS. Also, Sch A downregulated the expression of IL-8 and upregulated the expression of HO-1 mRNA in lung epithelial cells and cell supernatants, and resulted in the downregulation of the protein expression level of phosphorylated nuclear factor- $\kappa \mathrm{B}$.

Conclusions: Sch A inhibited the oxidative stress of lung epithelial cells induced by the combination of cigarette smoke extract and LPS. Sch A may be a potential therapeutic medication for COPD.

Keywords: Chronic obstructive pulmonary disease (COPD); cigarette smoke extract; lipopolysaccharide (LPS); oxidative stress; Schisandrin A (Sch A)

Submitted Aug 14, 2019. Accepted for publication Jan 07, 2020.

doi: $10.21037 /$ jtd.2020.01.24

View this article at: http://dx.doi.org/10.21037/jtd.2020.01.24

\section{Introduction}

Chronic obstructive pulmonary disease (COPD) is a chronic lung disease characterized by progressive, incompletely reversible airflow limitation and pulmonary inflammatory responses with recurrent and progressive development.
The acute exacerbation of COPD (AECOPD), an acute worsening of COPD, is characterized by worsening of respiratory symptoms, exceeding normal daily variability, and requires changes in medication therapy. The morbidity and mortality of COPD remain high to date. It is projected 
to be the third leading cause of death in the future (1). COPD not only significantly affects the quality of life but also results in a substantial economic burden (2). Despite numerous achievements, no effective drugs are available for managing COPD. Thus, effective medications for treating COPD are urgently needed.

The etiology and pathogenesis of AECOPD are not yet fully understood. The nuclear factor kappa B (NF-кB) signaling pathway has been implicated in $\operatorname{COPD}(3,4)$. $\mathrm{NF}-\kappa \mathrm{B}$ has a central role in the pathogenesis of chronic airway inflammatory diseases such as COPD. It is crucial in regulating inflammation and oxidative stress. It can regulate the activity of many cytokines, including interleukin (IL)- $1 \beta$, IL-6, IL-8, IL-12, tumor necrosis factor (TNF)- $\alpha$, monocyte chemotactic protein (MCP)-1, and adhesion molecules (5). Considering the previous findings on the $\mathrm{NF}-\kappa \mathrm{B}$ signaling pathway and the role of NF- $\mathrm{BB}$ in the pathogenesis of COPD, this study was performed to explore the mechanism underlying the role of NF- $\mathrm{KB}$ signaling pathway in the oxidative stress induced by the combination of cigarette smoke extract (CSE) and lipopolysaccharide (LPS) in lung epithelial cells.

Schisandrin A (Sch A), a natural lignan isolated from the herb Schisandra chinensis (Turcz.) Baill, has been reported to have diverse pharmacological activities, including anti-inflammatory, antioxidant, antibacterial, antiviral, and antitumor. It also improves immunity $(6,7)$. It has been shown to inhibit excessive proliferation and induce apoptosis in multiple cells. Wang et al. (8) showed that Sch A significantly reduced cell apoptosis and necrosis and increased cell survival in a primary culture of rat cortical neurons. Kong et al. (9) showed that Sch A increased cell viability and sensitized 5-fluorouracil (5-FU)-resistant HCT116 and SW480 cells to 5-FU. However, the protective effect of Sch A against lung oxidative stress induced by the combination of CSE and LPS remains unclear.

This study was performed to assess the protective effects of Sch A against oxidative stress induced by the combination of CSE and LPS in pulmonary epithelial cells and elucidate the potential mechanisms.

\section{Methods}

\section{Materials}

Sch A (purity $>98 \%$ ) was purchased from Chengdu Must Bio-Technology Co Ltd. (Sichuan, China). Antibodies specific for IL-8, heme oxygenase-1 (HO-1), and glyceraldehyde-3-phosphate dehydrogenase (GAPDH) were purchased from Shanghai Shenggong Biological Engineering Co, Ltd (Shanghai, China). A nuclear factor (NF)-кB Pathway Sampler Kit was purchased from Cell Signaling Technology Inc. (Shanghai, China).

\section{Cell culture}

Human lung epithelial cell line A549 was obtained with the courtesy of State Key Laboratory, Guangzhou Medical University. The cells were cultured in Dulbecco's modified Eagle's medium (DMEM), supplemented with $10 \%$ fetal bovine serum, $100 \mathrm{IU} / \mathrm{mL}$ penicillin, and $100 \mu \mathrm{g} / \mathrm{mL}$ streptomycin. They were incubated at $37^{\circ} \mathrm{C}$ in a humidified atmosphere of $5 \% \mathrm{CO}_{2}$. After reaching $70-80 \%$ confluence, the cells were subcultured for subsequent experiments.

\section{CSE preparation}

CSE derived from two cigarettes (Shuang X, Guangdong Zhong Yan Co. Ltd, Guangdong, China; $1.2 \mathrm{mg}$ nicotine, $11 \mathrm{mg}$ tar per cigarette) was filled slowly into a $50-\mathrm{mL}$ syringe and bubbled through $10 \mathrm{~mL}$ of DMEM. One cigarette yielded five draws of $50 \mathrm{~mL}$ with the syringe, with individual draws requiring approximately $10 \mathrm{~s}$ to complete. This preparation (100\% CSE) was titrated to $\mathrm{pH} 7.4$ and sterilized with a $0.22-\mathrm{mm}$ syringe filter. Serum-free cell culture medium was used to dilute $100 \%$ CSE to the working CSE concentrations. The final concentration was $4 \%$ for CSE (10) and $0.1 \mu \mathrm{g} / \mathrm{mL}$ for LPS (11).

\section{Evaluation of cell viability}

The 3-(4,5-dimethylthiazol-2-yl)-2,5-diphenyltetrazolium bromide (MTT) assay was used to examine cell viability. Lung epithelial cells $\left(5 \times 10^{3}\right.$ cells/well $)$ were cultured for $24 \mathrm{~h}$ in 96-well plates before treatment with the combination of $4 \%$ CSE and $0.1 \mu \mathrm{g} / \mathrm{mL}$ LPS or different concentrations of Sch A $(0,1,5,10,20,40$, and $60 \mu \mathrm{M})$. They were then incubated for $24 \mathrm{~h}$ at $37{ }^{\circ} \mathrm{C}$ in a humidified atmosphere containing $5 \% \mathrm{CO}_{2}$. Each well with MTT solution $(5 \mathrm{mg} / \mathrm{mL}, \mathrm{pH}$ 7.4) was further subjected to cultivation for another 4 h. Following the culture, the supernatant was carefully discarded, and $150 \mu \mathrm{L}$ of dimethyl sulfoxide was added to each well. The suspension was shaken for $10 \mathrm{~min}$, and the crystals were fully dissolved. A wavelength of $570 \mathrm{~nm}$ was selected, and the optical density (OD) was 
determined using a PE X5 microplate reader. The survival rate was calculated as OD for the treatment group/OD for the control group.

\section{Colorimetry for measuring the changes in antioxidant markers}

After culture, the cell supernatant was harvested. It was centrifuged at $1,000 \mathrm{rpm}$ for $10 \mathrm{~min}$ at $4{ }^{\circ} \mathrm{C}$ and stored at $-80{ }^{\circ} \mathrm{C}$. The levels of oxidative stress marker malondialdehyde (MDA) and anti-oxidant markers including superoxide dismutase (SOD), glutathione (GSH) were detected by colorimetry according to the instructions of the manufacturer(Jiancheng Company, Nanjing, China).

\section{Real-time-polymerase chain reaction}

Each group of cells was cultured for a certain time. The wells were then washed three times with precooled phosphate-buffered saline (PBS) (1×), followed by the addition of $1 \mathrm{~mL}$ of Trizol. An ultraviolet spectrophotometer was used to determine the concentration and purity of nucleic acids. cDNA was synthesized according to the instructions on the Prime Script RTreagent kit (Japan). Then, $2 \mu \mathrm{g}$ of total RNA was sampled for reverse transcription of full-length mRNA. Target mRNA quantification was performed by high productivity RTPCR in ViiATM 7 using a SYBR ${ }^{\circledR}$ Premix Ex Taq ${ }^{\mathrm{TM}}$ II Kit (Takara Biotechnology, Dalian, Liaoning, China) as per the manufacturer's protocol. The following primers were used: IL-8 sense: 5'-CCACCGGAGCACTCCATAAG-3'; antisense: 5'-GATGGTTCCTTCCGGTGGTT-3', HO-1 sense: 5'-AGTCTTCGCCCCTGTCTACT-3'; antisense: 5'-CTTCACATAGCGCTGCATGG-3', GAPDH sense: 5'-GAAAGCCTGCCGGTGACTAA-3'; antisense: 5'-AGGAAAAGCATCACCCGGAG-3'. The relative mRNA levels were determined using the $2^{-\Delta \Delta \mathrm{Ct}}$ method following normalization with the housekeeping gene GAPDH.

\section{Enzyme-linked immunosorbent assay}

The cell supernatant was harvested, centrifuged at $1,000 \mathrm{rpm}$ for $10 \mathrm{~min}$ at $4{ }^{\circ} \mathrm{C}$, and stored at $-80^{\circ} \mathrm{C}$. The concentration of IL- 8 and HO- 1 in the supernatant was detected using the enzyme-linked immunosorbent assay (ELISA) kit (Huamei Company, Wuhan, Hubei, China) following the manufacturer's protocols.

\section{Western blot analysis}

The cells in each group were cultured for a certain time and washed three times with ice-cooled PBS (1x). The RIPA (Radio Immunoprecipitation Assay) lysis buffer was added for treatment on ice, centrifuged at 12,000 rpm for $30 \mathrm{~min}$, and incubated at $4{ }^{\circ} \mathrm{C}$ for $20 \mathrm{~min}$. The supernatant was collected, and the concentration of protein was determined by the bicinchoninic acid method. Subsequently, $30 \mu \mathrm{g}$ of protein from each sample was separated using $10 \%$ sodium dodecyl sulphate-polyacrylamide gel electrophoresis, transferred onto polyvinylidene difluoride membranes (Millipore, Billerica, MA, USA), and then blocked with 5\% skim milk in a Tris-buffered saline- $0.1 \%$ Tween 20 buffer (TBST) for $1 \mathrm{~h}$. The membranes were then incubated with the corresponding primary antibodies against GAPDH(Cell Signaling Technology), Nuclear factor kappa beta (NF-кB) (Cell Signaling Technology), phosphorylated Nuclear factor kappa beta (pNF-кB) (Cell Signaling Technology), Inhibitor of NF- $\kappa \mathrm{B} \alpha(\mathrm{I} \kappa \mathrm{B} \alpha)$ (Cell Signaling Technology) and phosphorylated Inhibitor of NF- $\kappa \mathrm{B} \alpha(\mathrm{p}-\mathrm{I} \kappa \mathrm{B} \alpha)(\mathrm{Cell}$ Signaling Technology) antibody $(1: 2,000)$ at $4{ }^{\circ} \mathrm{C}$ overnight. Subsequently, the membranes were washed thrice with TBST (5 min each time) and incubated with a secondary antibody, at room temperature for $1 \mathrm{~h}$. An enhanced chemiluminescence detection system was used to detect the antibody-bound proteins on the membranes.

\section{Statistical analysis}

At least three samples were required for experiments. The data were presented as mean \pm standard error of mean. Data were statistically compared using one-way analysis of variance and statistically analyzed with GraphPad Prism 5.0. $\mathrm{P}$ values less than 0.05 were considered statistically significant.

\section{Results}

\section{Effect of Sch A on the growth of lung epithelial cells induced by the combination of CSE and LPS}

Lung epithelial cells were treated with different concentrations of Sch A (1-60 $\mu \mathrm{mol} / \mathrm{L})$ for $48 \mathrm{~h}$ to investigate the inhibitory effect of Sch A on lung epithelial cells. Different concentrations of Sch A could inhibit the proliferation of lung epithelial cells in a dose-dependent manner (Figure 1A). The cells were treated with a combination of $4 \%$ CSE and $0.1 \mu \mathrm{g} / \mathrm{mL}$ LPS for $24 \mathrm{~h}$ 
A

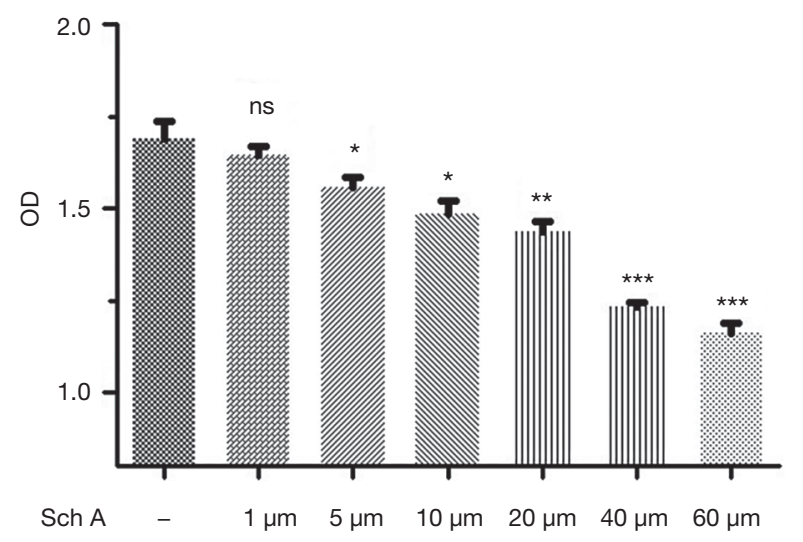

B

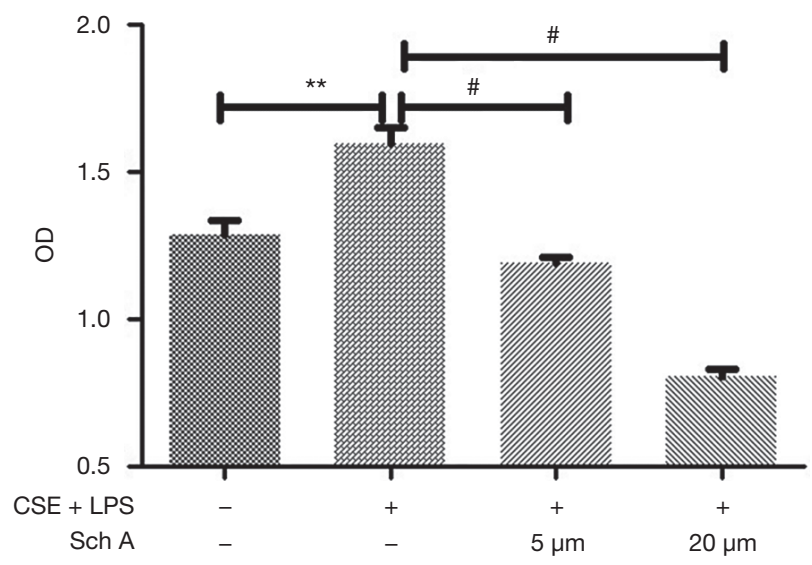

Figure 1 Effect of SchA on the growth of lung epithelial cells induced by CSE combined with LPS. (A) Lung epithelial cells were exposed

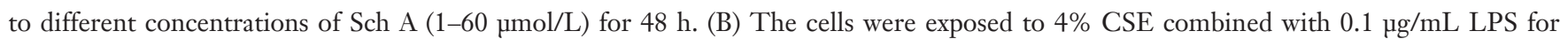
$24 \mathrm{~h}$, followed by exposure to 5 or $20 \mu \mathrm{M}$ Sch A for $48 \mathrm{~h}$. Cell viability was detected using the MTT assay. Data were presented as the mean \pm standard error of mean (SEM) of three independent experiments (ratio of treated samples to control samples). ns, no statistical difference. ${ }^{*}, * * \mathrm{P}<0.05 ;{ }^{* * *} \mathrm{P}<0.01 ;{ }^{*} \mathrm{P}<0.01$. CSE, cigarette smoke extract; LPS, lipopolysaccharide; MTT, 3-(4,5-dimethylthiazol-2-yl)-2,5diphenyltetrazolium bromide.

to establish an acute exacerbation model of COPD. The results showed that the combination of CSE and LPS could significantly promote the proliferation of lung epithelial cells compared with the control group. The addition of 5 and $20 \mu \mathrm{M}$ Sch A reverted the abnormal proliferation of lung epithelial cells conferred by the combination of $4 \%$ CSE and $0.1 \mu \mathrm{g} / \mathrm{mL}$ LPS $(\mathrm{P}<0.05$ compared with the control group, Figure 1B). These results suggested that Sch A ameliorated the abnormal proliferation of lung epithelial cells induced by the combination of CSE and LPS.

\section{Effects of Sch $A$ on the levels of antioxidant markers in lung epithelial cells induced by the combination of CSE and LPS}

Sch A could significantly inhibit the proliferation of lung epithelial cells in an in vitro model of AECOPD. Next, the levels of MDA, SOD, and GSH were examined in the cell supernatant of lung epithelial cells. As shown in Figure 2, the combination of CSE and LPS induced high levels of MDA and low levels of SOD and GSH. The changes were statistically significant compared with the control group $(\mathrm{P}<0.05)$. Treatment of lung epithelial cells with 5 or $20 \mu \mathrm{M}$ Sch A significantly decreased the level of MDA and increased the levels of SOD and GSH compared with the model group (all $\mathrm{P}<0.05)$.

\section{Effects of Sch A on oxidative stress-related gene expression in lung epithelial cells induced by the combination of CSE and LPS}

Sch A could significantly affect the antioxidant index of AECOPD in in vitro models. Next, the effects of Sch A on oxidative stress-related gene expression were examined. As shown in Figure 3, the combination of CSE and LPS significantly upregulated the expression of IL-8 and HO-1 mRNA in lung epithelial cells compared with the control group (both $\mathrm{P}<0.05$ ). The expression of IL- 8 and HO-1 was also detected in the supernatant of lung epithelial cells by performing ELISA. Consistent with the PCR results, the combination of CSE and LPS significantly upregulated the expression of IL-8 and HO-1 in lung epithelial cells compared with the control group (both $\mathrm{P}<0.05$ ). Treatment of lung epithelial cells with 5 or $20 \mu M$ Sch A markedly decreased the expression and secretion of IL-8 compared with the model group (both $\mathrm{P}<0.05$ ). Compared with the model group, there was no statistical difference in the $5 \mu \mathrm{M}$ dose of Sch A group, only $20 \mu \mathrm{M}$ dose of Sch A had 
A
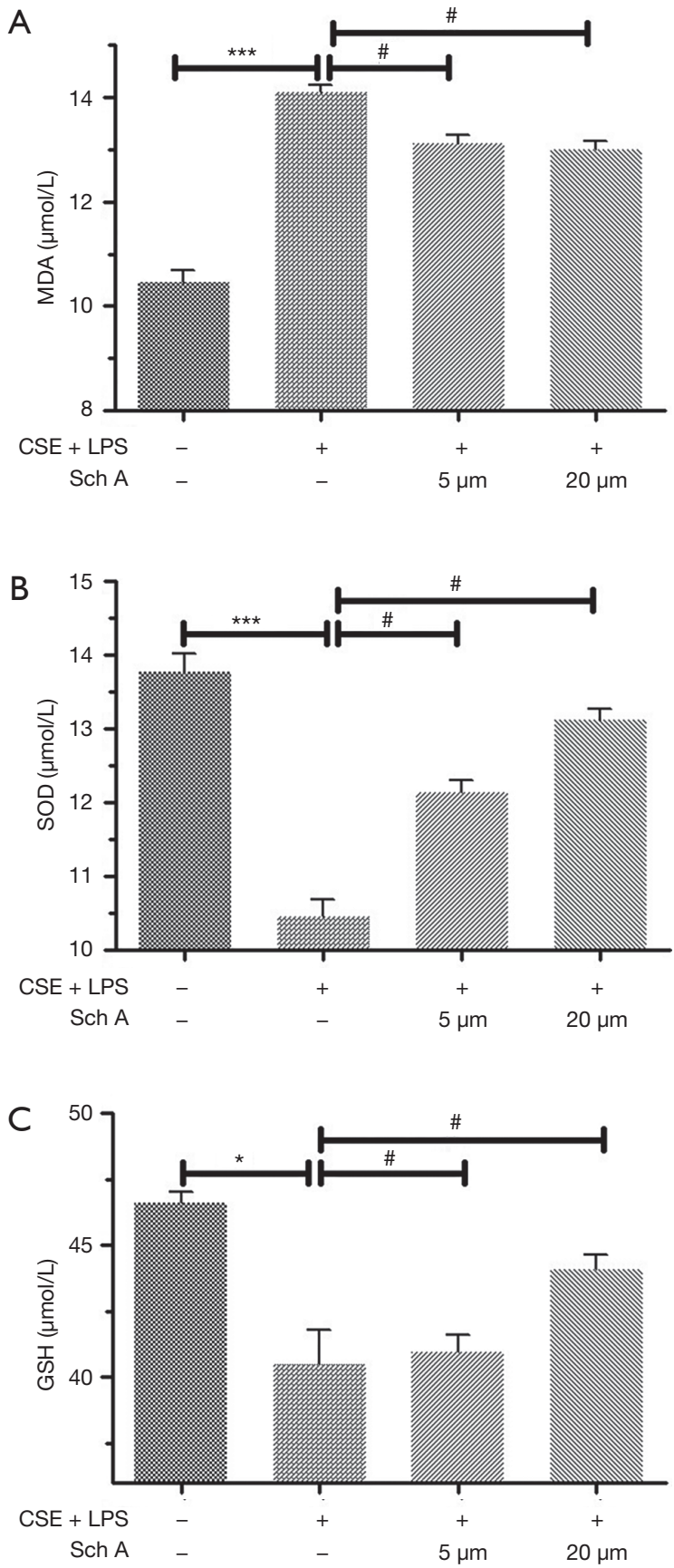

Figure 2 Effects of Sch A on the levels of anti-oxidant markers in lung epithelial cells induced by CSE combined with LPS. The cells were pretreated with CSE combined with LPS for $24 \mathrm{~h}$, followed by exposure to 5 or $20 \mu \mathrm{M}$ Sch A for $48 \mathrm{~h}$. Next, the supernatant was used to detect the levels of MDA (A), SOD (B) and GSH (C). Data were represented as the mean \pm SEM of three independent experiments. ${ }^{*} \mathrm{P}<0.05$; ${ }^{* * *} \mathrm{P}<0.01 ;{ }^{\#} \mathrm{P}<0.05$. CSE, cigarette smoke extract; LPS, lipopolysaccharide; MDA, malondialdehyde; SOD, superoxide dismutase; GSH, glutathione. significant up-regulation of HO-1 expression $(\mathrm{P}<0.05)$. The results of PCR and ELISA indicated that Sch A could inhibit the expression of oxidative stress-related genes.

Effects of Sch $A$ on the oxidative stress-related signaling pathway in lung epithelial cells induced by the combination of CSE and LPS

This study further explored the intracellular signaling pathways involved in the inhibitory effect of Sch A on the production of oxidative stress-related genes. As shown in Figure 4, the combination of CSE and LPS upregulated the expression of NF- $\mathrm{\kappa B}$ signaling-related protein in lung epithelial cells, suggesting that the combination might induce AECOPD by activating the NF- $\mathrm{\kappa B}$ signaling pathway. In contrast, in AECOPD induced by the combination, Sch A inhibited the expression of NF- $\kappa \mathrm{B}$ signaling-related proteins. Hence, Sch A exerted the protective effect against AECOPD possibly via the NF- $\mathrm{KB}$ signaling pathway.

\section{Discussion}

The in vitro AECOPD model was established in this study by administering the combination of CSE and LPS (12). Cigarette smoke contains a toxic mixture of nicotine, nitric oxide, and formaldehyde, which accumulates in the alveolar cells, leading to the release of a variety of inflammatory mediators and cytokines that cause injury to the airways and further promote the development of inflammatory responses. LPS is one of the main components of Gramnegative bacterial endotoxin. It can stimulate neutrophils and macrophages to release a large number of cytokines and inflammatory mediators. Tatiana et al. (13) showed that the combination of CSE and LPS enhanced the release and expression of chemokines, such as IL-8, MCP-1, and Gro- $\alpha$, in A549 cells, which might be related to the activation of ERK1/2 and JAK/STAT signaling pathways. In this study, a well-recognized in vitro model was used. The study confirmed that the combination of CSE and LPS could interfere with the proliferation of lung epithelial cells, indicating that the establishment of the AECOPD model was successful.

Oxidative stress is caused by the excessive production of oxidants and/or reduction in anti-oxidants, resulting in oxidative-anti-oxidative imbalance. Excess oxidants can lead to tissue or organ injury. Oxidative stress not only directly damages lung tissues but also results in the persistence of 
A
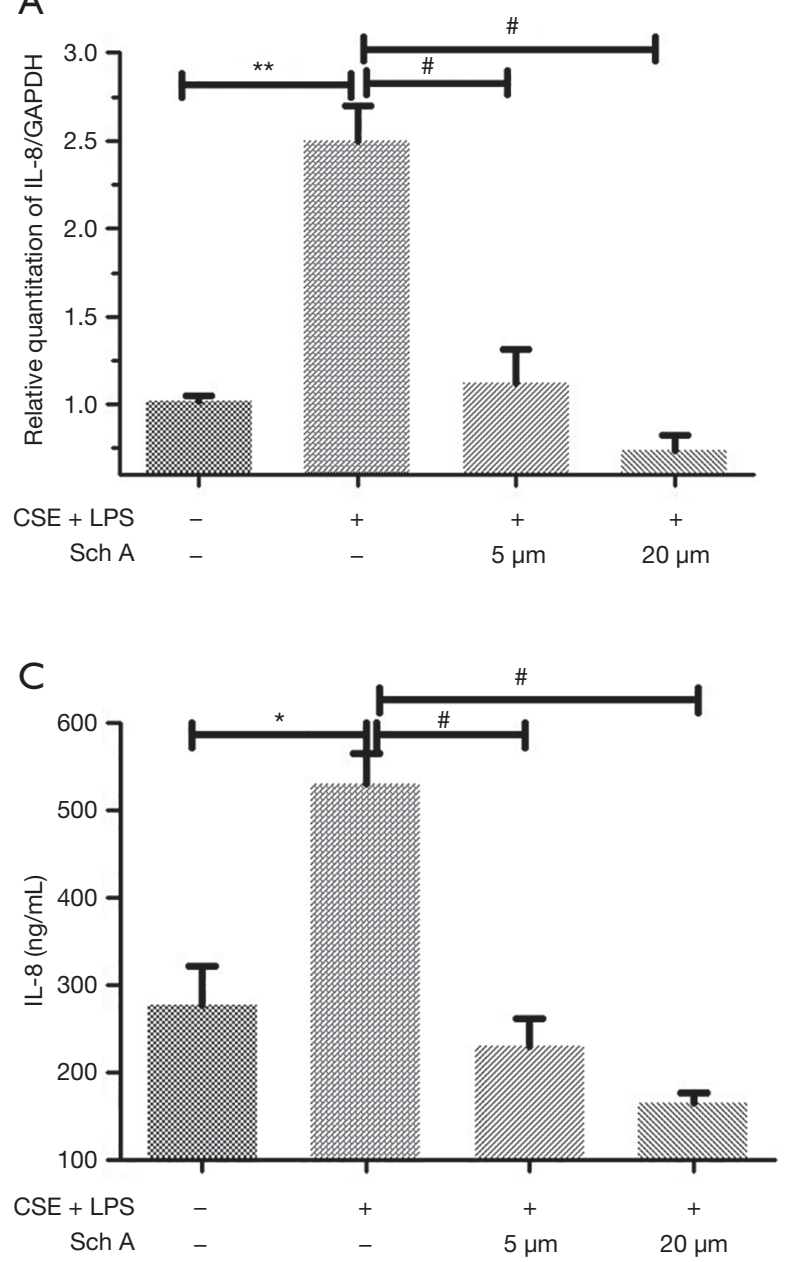

B

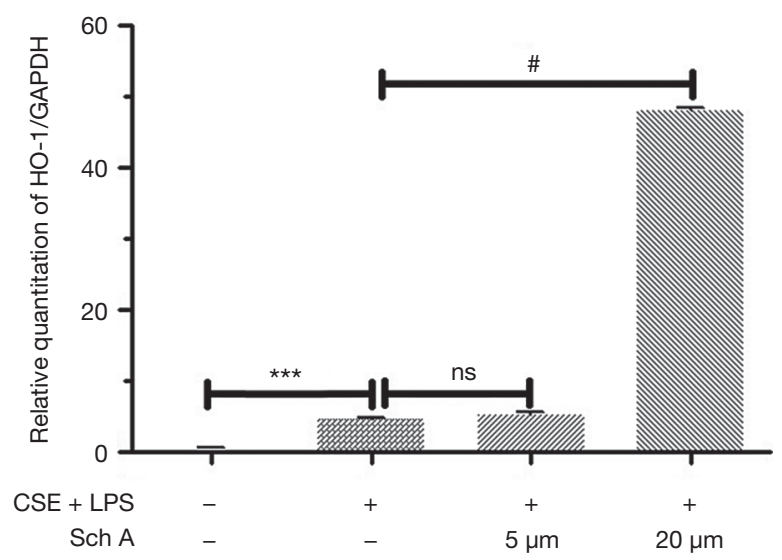

D

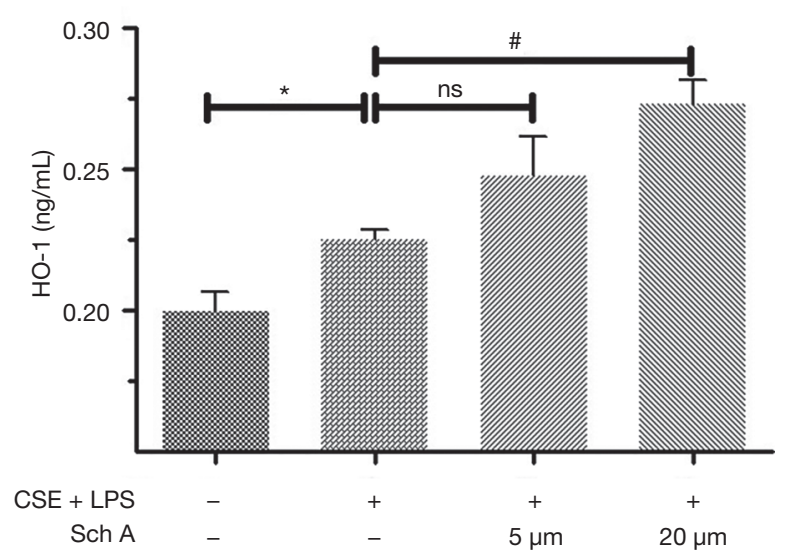

Figure 3 Effects of SchA on oxidative stress-related gene expression in lung epithelial cells induced by CSE combined with LPS. The cells were pretreated with CSE combined with LPS for $24 \mathrm{~h}$, followed by exposure to 5 or $20 \mu \mathrm{M}$ Sch A for 48 h. Expressions levels of IL-8 (A) and HO-1 (B) mRNA were measured using qRT-PCR. Expression levels of IL-8 (C) and HO-1 (D) were measured using ELISA. Data were presented as the mean \pm SEM of three independent experiments. ${ }^{*}{ }^{* *} \mathrm{P}<0.05$; ${ }^{* * *} \mathrm{P}<0.01$; ${ }^{*} \mathrm{P}<0.05$. CSE, cigarette smoke extract; LPS, lipopolysaccharide; ELISA, enzyme-linked immunosorbent assay.

oxidative stress. This further promotes the chemotaxis of inflammatory cells, increases the expression of inflammatory mediators, and inactivates the protease-oxidation processes, thereby promoting the development of COPD (14). MDA is one of the representative products of lipid peroxidation that can reflect the severity of oxidative stress. The level of MDA indirectly reflects the severity of cell injury by free radicals (15). SOD is a ubiquitous enzyme that can scavenge oxygen free radicals and prevent cells from nuclear oxidative cleavage. SOD is an important antioxidant whose activity represents the total antioxidant capacity (16). GSH is a nonprotein tripeptide containing sulfhydryl groups, which can resist endogenous and exogenous oxidation, remove excess reactive oxygen free radicals in the body, and prevent cells from further oxidative reactions (17). In this study, the level of MDA significantly increased in the model group, whereas SOD and GSH levels were significantly lower than those in the control group, suggesting an imbalance of oxidation/antioxidation in AECOPD. The levels of MDA markedly decreased and the levels of SOD and GSH significantly increased after administering different concentrations of Sch A. The results showed that the therapeutic effect of Sch A on the AECOPD cell model in vitro might be related to the improvement in antioxidant 


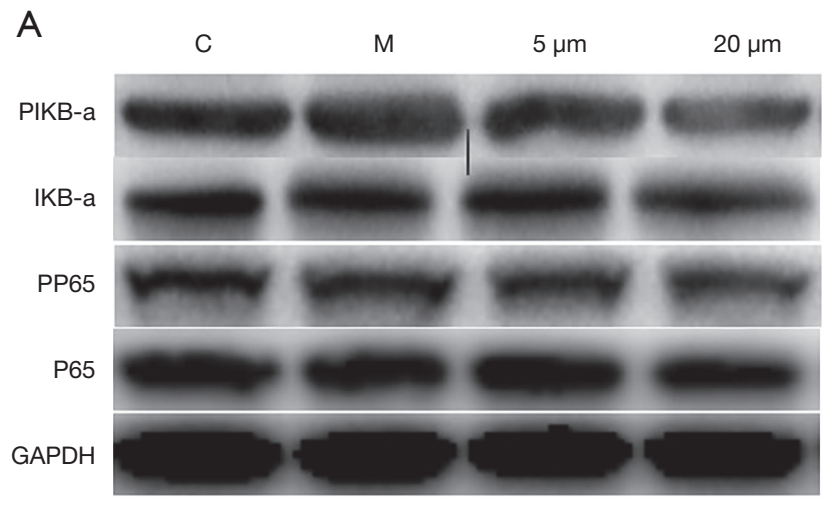

B

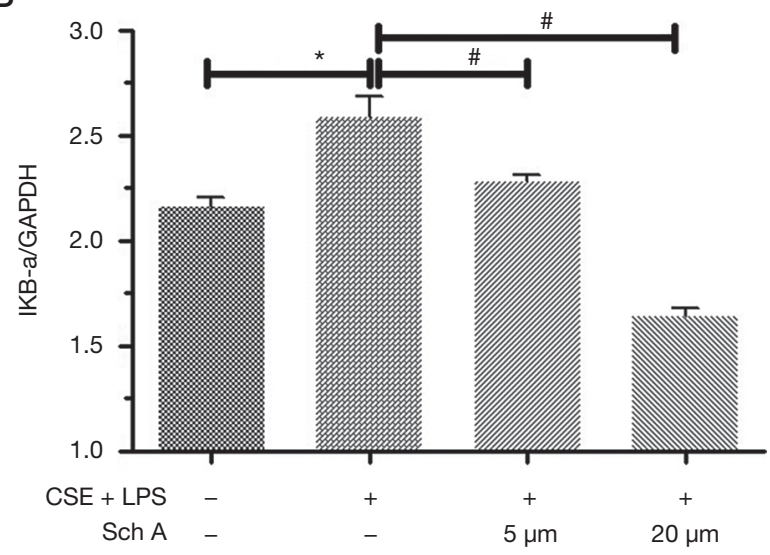

C

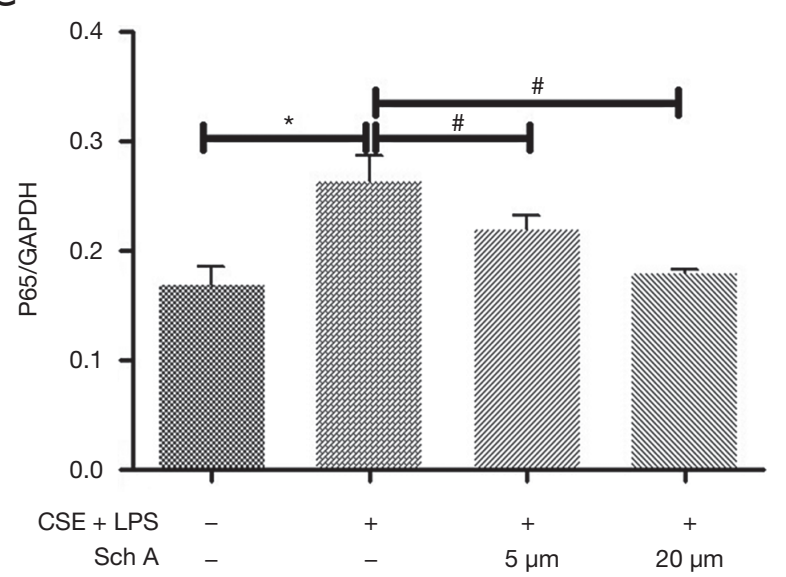

Figure 4 Effects of SchA on oxidative stress-related signaling pathway in lung epithelial cells induced by CSE combined with LPS. The cells were pretreated with CSE combined with LPS for $24 \mathrm{~h}$, followed by exposure to 5 or $20 \mu \mathrm{M}$ Sch A for $48 \mathrm{~h}$. Data were presented as mean \pm SEM of three independent experiments. ${ }^{*} \mathrm{P}<0.05 ;{ }^{*} \mathrm{P}<0.05$. CSE, cigarette smoke extract; LPS, lipopolysaccharide. activity.

Wang et al. (18) found Resveratrol can treat experimental COPD in rats by down regulating MDA, up regulating SOD expression, and decreased serum levels of IL-6 and IL-8. Li et al. (19) also found that the protective effect of methylallyl sulfonate (AMSO2) on experimental lung injury induced by CSE was related to reducing of MDA, enhancing activities of SOD, and inhibiting NF- $\kappa \mathrm{B}$ pathway. And these are basically consistent with our research results.

COPD is a chronic inflammation of bronchi, lung parenchyma, and pulmonary vasculature. Several inflammatory cells and mediators are involved in the pathogenesis of COPD. The main cytokines associated with the pathogenesis of COPD include IL- $1 \beta$, IL-6, IL-8, and TNF- $\alpha$ (20). Of these, IL- 8 is considered as an important cytokine involved in the development of airway inflammation in COPD (21). IL-8 is a chemokine that recruits and activates neutrophils and $\mathrm{T}$ lymphocytes, induces morphological changes in neutrophils, and causes a transient increase in intracellular calcium concentrations. High levels of TNF- $\alpha$ lead to degranulation, release of lysosomal enzymes, upregulation of adhesion proteins, formation of bioactive lipids, and a respiratory burst of superoxide and lysosomal enzymes, thereby promoting chronic airway inflammation. HO-1 is an enzyme that can be induced under stress conditions, such as ischemia, hypoxia, and shock, to counteract oxidative stress and protect cells from damage (22). This study showed that the level of HO-1 significantly increased after administering the combination of CSE and LPS. The level of HO-1 markedly improved after Sch A treatment. Vanella et al. (23) results also showed that a new antioxidants formulation can also play a protective role in COPD cell model in vitro by up regulating the expression of $\mathrm{HO}-1$, which is consistent with our results of Sch A.

$\mathrm{NF}-\mathrm{\kappa B}$ is a nuclear protein factor that specifically binds to the immunoglobulin $\mathrm{\kappa B}$ light chain gene enhancer. It has an important role in the development of COPD. It is central to multiple signaling pathways and essential in regulating inflammation and oxidative stress. After activation, NF- $\kappa B$ can regulate a variety of transcription factors associated with cell growth, survival, and apoptosis. It participates in the development of inflammatory response, immune response, proliferation, apoptosis, and other physiological activities of cells (3).

Normally, NF- $\kappa \mathrm{B}$ is a dimeric structure that binds to one of the three typical NF- $\mathrm{NB}$ inhibitory proteins or 
exists in the form of precursor proteins $\mathrm{P} 105$ and $\mathrm{P} 100$. Being inactive in the cytoplasm, I $\kappa \mathrm{B}$ is a specific inhibitor protein inactive in the cytoplasm. It covers the nuclear translocation signal of $\mathrm{NF}-\mathrm{\kappa B}$, thereby preventing the entry of NF- $\mathrm{NB}$ into the nucleus, DNA binding, transcription, and expression. Stress factors such as viruses, bacteria, cytokines, growth factors, and platelet-activating factors, and chemotherapeutic agents can cause the activation and phosphorylation of IKK complexes and ubiquitination and degradation of IкB under the action of proteolytic enzymes, releasing P50/P65. The released P50/P65 nuclear ectopic site exposes the nuclear localization signal region on NF- $\mathrm{KB}$, which specifically binds to the gene's $\kappa \mathrm{B}$ site and is thus activated (24). Plasma NF- $\kappa \mathrm{B}$ levels of the COPD patients were significantly higher than those of the control subjects $(\mathrm{P}<0.001)(25)$. The results showed that $\mathrm{I} \kappa \mathrm{B} \alpha$ phosphorylation further decreased under the stimulation of the combination of CSE and LPS, while Sch A inhibited the phosphorylation and degradation of $I \kappa B \alpha$. The transcriptional activity of NF- $\mathrm{\kappa B}$ significantly increased and the transcriptional activity of NF- $\kappa \mathrm{B}$ gradually decreased after treatment with different concentrations of Sch A. Therefore, Sch A could inhibit the nuclear translocation of NF- $\mathrm{KB}$ induced by the combination of CSE and LPS in lung epithelial cells by inhibiting the phosphorylation and degradation of IкB $\alpha$ in the cytoplasm.

Sch A is one of the main active components of Schisandra chinensis. In addition to Sch A, other active components or active parts of Schisandra chinensis, including ethanol extract (EE) and ethanol-water extract (EWE) of S. chinensis, and Schisandrin $\mathrm{B}$, were found to have protective effects on inflammatory lung injury (26-28). Our study has a certain significance to further clarify the mechanism of Schisandra in the treatment of "cough deficiency asthma" and to clarify the material basis of its effect.

In conclusion, Sch A exerted protective and therapeutic effects against oxidative stress induced by CSE combined with LPS, including SchA can significantly inhibit cell proliferation, improve the expression of antioxidant markers, inhibit the expression of IL-8, and significantly up regulate the expression of HO-1 in some doses, possibly through the NF- $\kappa$ B signaling pathway. The findings suggested that Sch A might be a potential therapeutic option for AECOPD.

\section{Acknowledgments}

Funding: This study was supported by the National
Natural Science Foundation of China (No. 81202674 and 81774103).

\section{Footnote}

Conflicts of Interest: The authors have no conflicts of interest to declare.

Ethical Statement: The authors are accountable for all aspects of the work in ensuring that questions related to the accuracy or integrity of any part of the work are appropriately investigated and resolved.

Open Access Statement: This is an Open Access article distributed in accordance with the Creative Commons Attribution-NonCommercial-NoDerivs 4.0 International License (CC BY-NC-ND 4.0), which permits the noncommercial replication and distribution of the article with the strict proviso that no changes or edits are made and the original work is properly cited (including links to both the formal publication through the relevant DOI and the license). See: https://creativecommons.org/licenses/by-nc-nd/4.0/.

\section{References}

1. Adeloye D, Chua S, Lee C, et al. Global and regional estimates of COPD prevalence: Systematic review and meta-analysis. Journal of Global Health 2015;5:020415.

2. Wang C, Xu J, Yang L, et al. Prevalence and risk factors of chronic obstructive pulmonary disease in China (the China Pulmonary Health $[\mathrm{CPH}]$ study): a national cross-sectional study. Lancet 2018;391:1706-17.

3. Zhang Y, Li Y, Shi C, et al. Angiotensin-(1-7)-mediated Mas1 receptor/NF-kB-p65 signaling is involved in a cigarette smoke-induced chronic obstructive pulmonary disease mouse model. Environ Toxicol 2018;33:5-15.

4. Xu H, Sun Q, Lu L, et al. MicroRNA-218 acts by repressing TNFR1-mediated activation of NF- $\mathrm{KB}$, which is involved in MUC5AC hyper-production and inflammation in smoking-induced bronchiolitis of COPD. Toxicology Letters 2017;280:171.

5. Compagno M, Wei KL, Grunn A, et al. Mutations of multiple genes cause deregulation of NF-kB in diffuse large B-cell lymphoma. Nature 2009;459:717-21.

6. Kwon DH, Cha HJ, Choi EO, et al. Schisandrin A suppresses lipopolysaccharide-induced inflammation and oxidative stress in RAW 264.7 macrophages by suppressing the NF-kB, MAPKs and PI3K/Akt pathways and activating 
Nrf2/HO-1 signaling. International Journal of Molecular Medicine 2018;41:264-74.

7. Song F, Zeng K, Liao L, et al. Schizandrin A Inhibits Microglia-Mediated Neuroninflammation through Inhibiting TRAF6-NF- $\kappa$ B and Jak2-Stat3 Signaling Pathways. PLoS One 2016;11:e0149991.

8. Wang CP, Li GC, Shi Y W, et al. Neuroprotective effect of schizandrin A on oxygen and glucose deprivation/ reperfusion-induced cell injury in primary culture of rat cortical neurons. J Physiol Biochem 2014;70:735-47.

9. Kong D, Zhang D, Chu X, et al. Schizandrin A enhances chemosensitivity of colon carcinoma cells to 5-fluorouracil through up-regulation of miR-195. Biomed Pharmacother 2018;99:176-83.

10. Wang C, Ding H, Tang X, Effect of Liuweibuqi Capsules in Pulmonary Alveolar Epithelial Cells and COPD Through JAK/STAT Pathway. Cell Physiol Biochem 2017;43:743-56.

11. Liu N, Zheng JX, Zhuang YS, et al. Anti-Inflammatory Effects of Schisandrin B on LPS-Stimulated BV2 Microglia via Activating PPAR- $\gamma$. Inflammation 2017;40:1006-11.

12. D'Anna C, Cigna D, Sano CD, et al. Exposure to cigarette smoke extract and lipopolysaccharide modifies cytoskeleton organization in bronchial epithelial cells. Exp Lung Res 2017;43:347.

13. Victoni T, Gleonnec F, Lanzetti M, et al. Roflumilast N-Oxide Prevents Cytokine Secretion Induced by Cigarette Smoke Combined with LPS through JAK/STAT and ERK1/2 Inhibition in Airway Epithelial Cells. PLoS One 2014;9:e85243.

14. Grady ST, Koutrakis P, Hart JE, et al. Indoor black carbon of outdoor origin and oxidative stress biomarkers in patients with chronic obstructive pulmonary disease. Environ Int 2018;115:188-95.

15. Zhuan B, Yu Y, Yang Z, et al. Mechanisms of oxidative stress effects of the NADPH oxidase-ROS-NF- $\mathrm{B}$ transduction pathway and VPO1 on patients with chronic obstructive pulmonary disease combined with pulmonary hypertension. Eur Rev Med Pharmacol Sci 2017;21:3459.

16. Bajpai J, Prakash V, Kant S, et al. Study of oxidative stress biomarkers in chronic obstructive pulmonary disease and their correlation with disease severity in north Indian population cohort. Lung India 2017;34:324.

17. Beigi Harchegani A, Tahmasbpour E, Borna H, et al. Free Radical Production and Oxidative Stress in Lung Tissue of Patients Exposed to Sulfur Mustard: An Overview of Cellular and Molecular Mechanisms. Chem Res Toxicol 2018;31:211-22.

18. Wang XL, Li T, Li JH, et al. The Effects of Resveratrol on
Inflammation and Oxidative Stress in a Rat Model of Chronic Obstructive Pulmonary Disease. Molecules 2017;22:1529.

19. Li A, Liu Y, Zhu X, et al. Methylallyl sulfone attenuates inflammation, oxidative stress and lung injury induced by cigarette smoke extract in mice and RAW264.7 cells. Int Immunopharmacol 2018;59:369-74.

20. Silva BS, Lira FS, Rossi FE, et al. Inflammatory and Metabolic Responses to Different Resistance Training on Chronic Obstructive Pulmonary Disease: A Randomized Control Trial. Front Physiol 2018;9:262.

21. Lee KH, Lee CH, Woo J, et al. Cigarette Smoke Extract Enhances IL-17AInduced IL-8 Production via UpRegulation of IL-17R in Human Bronchial Epithelial Cells. Mol Cells 2018;41:282-9.

22. Lee KH, Jeong J, Koo YJ, et al. Exogenous neutrophil elastase enters bronchial epithelial cells and suppresses cigarette smoke extract-induced heme oxygenase- 1 by cleaving sirtuin 1. J Biol Chem 2017;292:11970-9.

23. Vanella L, Li VG, Distefano A, et al. A new antioxidant formulation reduces the apoptotic and damaging effect of cigarette smoke extract on human bronchial epithelial cells. Eur Rev Med Pharmacol Sci 2017;21:5478-84.

24. Deo P, Cheng ZZ, Han WZ, et al. The Change and Significance of Nuclear Transcription Factor NF-kB at Different Period in Mice While Gradually Develop COPD. Progress in Modern Biomedicine 2011;11:3219-26.

25. Uysal P, Simsek G, Durmus S, et al. Evaluation of Plasma Antimicrobial Peptide LL-37 and Nuclear factor-kappaB Levels in Stable Chronic Obstructive Pulmonary Disease. Int J Chron Obstruct Pulmon Dis 2019;14:321-30.

26. Jia R, Zhang H, Yang Z, et al. Protective effects of Schisandrin $\mathrm{B}$ on cigarette smoke-induced airway injury in mice through Nrf2 pathway. Int Immunopharmacol 2017;53:11-6.

27. Zhong S, Liu XD, Nie YC, et al. Antitussive activity of the Schisandra chinensis fruit polysaccharide (SCFP-1) in guinea pigs models. J Ethnopharmacol 2016;194:378-85.

28. Zhong S, Nie YC, Gan ZY, et al. Effects of Schisandra chinensis extracts on cough and pulmonary inflammation in a cough hypersensitivity guinea pig model induced by cigarette smoke exposure. J Ethnopharmacol 2015;165:73-82.

Cite this article as: Zhang $\mathrm{D}, \mathrm{Hu} \mathrm{S}, \mathrm{Li} \mathrm{W}$, Ao R, Wu Z, Zhang Z, Han L. Schisandra A ameliorates cigarette smoke extract and lipopolysaccharide-induced oxidative stress in lung epithelial cells. J Thorac Dis 2020;12(3):394-402. doi: $10.21037 /$ jtd.2020.01.24 